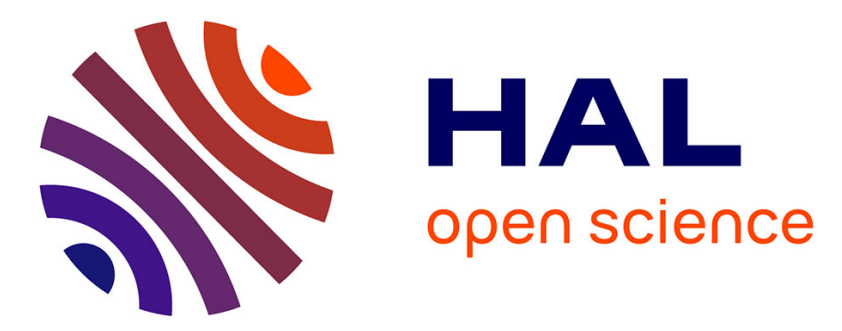

\title{
Casimir energy for two and three superconducting coupled cavities: numerical calculations
}

\author{
L. Rosa, S. Avino, E. Calloni, S. Caprara, M. de Laurentis, R. de Rosa,
} Giampiero Esposito, M. Grilli, E. Majorana, G. P. Pepe, et al.

\section{- To cite this version:}

L. Rosa, S. Avino, E. Calloni, S. Caprara, M. de Laurentis, et al.. Casimir energy for two and three superconducting coupled cavities: numerical calculations. The European Physical Journal Plus, 2017, 132 (11), pp.478. 10.1140/epjp/i2017-11750-y · hal-01476557

\section{HAL Id: hal-01476557 \\ https://hal.science/hal-01476557}

Submitted on 28 Apr 2018

HAL is a multi-disciplinary open access archive for the deposit and dissemination of scientific research documents, whether they are published or not. The documents may come from teaching and research institutions in France or abroad, or from public or private research centers.
L'archive ouverte pluridisciplinaire HAL, est destinée au dépôt et à la diffusion de documents scientifiques de niveau recherche, publiés ou non, émanant des établissements d'enseignement et de recherche français ou étrangers, des laboratoires publics ou privés. 


\section{Casimir energy for two and three superconducting coupled cavities}

L. Rosa ${ }^{1,2}$, S. Avino ${ }^{2,3}$, E. Calloni ${ }^{1,2}$, S. Caprara ${ }^{4,5}$, M. De Laurentis ${ }^{1}$, R. De Rosa ${ }^{1,2}$, Giampiero Esposito 2 , M. Grilli, ${ }^{4,5}$, E. Majorana ${ }^{6}$, G. P. Pepe ${ }^{7}$, S. Petrarca ${ }^{4,6}$, P. Puppo ${ }^{6}$, P. Rapagnani $^{4,6}$, F. Ricci ${ }^{4,6}$, C. Rovelli ${ }^{8}$, P. Ruggi ${ }^{9}$, N. L. Saini ${ }^{4}$, C. Stornaiolo ${ }^{2}$, F. Tafuri ${ }^{1}$

1 Università di Napoli Federico II, Dipartimento di Fisica "Ettore Pancini", Complesso Universitario di Monte S. Angelo, Via Cintia Edificio 6, 80126 Napoli, Italy

${ }^{2}$ INFN Sezione di Napoli, Complesso Universitario di Monte S. Angelo, Via Cintia Edificio 6, 80126 Napoli, Italy

3 INO-CNR, Comprensorio Olivetti, Via Campi Flegrei 34-80078 Pozzuoli (NA), Italy

4 Università di Roma "La Sapienza",

P.le A. Moro 2, I-00185, Roma, Italy

${ }^{5}$ ISC-CNR and Consorzio Nazionale Interuniversitario per le Scienze Fisiche della Materia (CNISM),

Unità di Roma "La Sapienza", P.le A. Moro 2, I-00185, Roma, Italy

${ }^{6}$ INFN Sezione di Roma, P.le A. Moro 2, I-00185, Roma, Italy

7 Università di Napoli Federico II, Dipartimento di Fisica "Ettore Pancini", piazzale Tecchio 80, 80126 Napoli, Italy

8 Aix Marseille Université CNRS, CPT, UMR 7332, 13288 Marseille, France

Universitè de Toulon, CNRS, CPT, UMR 7332, 83957 La Garde, France and

${ }^{9}$ European Gravitational Observatory (EGO), I-56021 Cascina (Pi), Italy 


\begin{abstract}
In this paper we study the behavior of the Casimir energy of a "multi-cavity" across the transition from the metallic to the superconducting phase of the constituting plates. Our analysis is carried out in the framework of the ARCHIMEDES experiment, aiming at measuring the interaction of the electromagnetic vacuum energy with a gravitational field. For this purpose it is foreseen to modulate the Casimir energy of a layered structure composing a multi-cavity coupled system by inducing a transition from the metallic to the superconducting phase. This implies a thorough study of the behavior of the cavity, where normal metallic layers are alternated with superconducting layers, across the transition. Our study finds that, because of the coupling between the cavities, mainly mediated by the transverse magnetic modes of the radiation field, the variation of energy across the transition can be very large.
\end{abstract}

PACS numbers: 12.20.Ds, 12.20.-m, 74.25.-q, 74.78.Fk 


\section{INTRODUCTION}

The ARCHIMEDES experiment [1] is designed for testing whether the energy of vacuum fluctuations, foreseen by quantum electrodynamics, contributes to gravity, through the coupling demanded by quantum field theory in curved spacetime [2 [5], where the Einstein tensor is taken to be proportional to the expectation value of the regularized and renormalized energy-momentum tensor of matter fields. The idea is to weigh the vacuum energy stored in a rigid Casimir cavity made by parallel conducting plates, by modulating the reflectivity of the plates upon inducing a transition from the metallic to the superconducting phase [1]. In order to enhance the effect, a multilayer cavity is considered, obtained by superimposing many cavities. This structure is natural in the case of crystals of type-II superconductors, particularly cuprates, being composed by $\mathrm{Cu}-\mathrm{O}$ planes, that undergo the superconducting transition, separated by nonconducting planes. A crucial aspect to be tested is thus the behavior of the Casimir energy [6] for a multi-cavity when the layers undergo the phase transition from the metallic to the superconducting phase. Until now only the case of a cavity having a single layer that undergoes the superconducting transition was considered, the other reflecting plate being just metallic (not superconducting), in Refs. [7, 8]. The generalization to the case of a system of coupled superconducting layered cavities is still lacking. With respect to the ARCHIMEDES project the main goal is to study the possibility of enhancing the modulation factor $\eta=\frac{\Delta E_{\text {cas }}}{E_{\text {cas }}}$ were $\Delta E_{\text {cas }}$ is the difference of Casimir energy in normal and superconducting states. The value obtained in Ref. [7, 8], considering a cavity with a single superconducting layer and a transition temperature of about $1 \mathrm{~K}$ is $\eta_{l} \approx 10^{-8}$. This value was compliant with a previous experiment devoted to ascertain the vacuum energy contribution to the total condensation energy [9, 10, but it is not sufficient to prove the weight of the vacuum, because it is in absolute too small. It is therefore necessary to consider high- $T_{c}$ superconductors where condensation energy is much higher and also the absolute value of vacuum energy variation is expected to be correspondingly larger.

On the other hand, in Ref. [11], considering a cavity based on a high- $T_{c}$ layered superconductor, a factor as high as $\eta_{h}=4 \cdot 10^{-4}$ has been estimated, under the approximation of flat plasma sheets at zero temperature, no conduction in normal state (here $E_{\text {cas }}$ is the energy of the ideal cavity) and charge density of $n=10^{14} \mathrm{~cm}^{-2}$. The ARCHIMEDES sensitivity is expected to be capable of ascertain the interaction of gravity and vacuum energy also for values lower than $\eta_{h}=4 \cdot 10^{-4}$, up to $1 / 100$ of this value [1]. Clearly it is important to understand more firmly if dealing with layered superconducting structures the modulation depth can be sufficiently high. This is the study of the present paper. Considering in particular the multi-layer cavity, the general assumption adopted so far has been that the Casimir energy obtained by overlapping many cavities is the sum of the energies of each individual cavity. This is true if the distances between neighboring cavities are large (in the sense that the

thickness of each metallic layer separating the various cavities is very large with respect to the penetration depth of the radiation field). Of course, this is no longer true if the thickness of these metallic inter-cavity layers gets thinner and thinner. The evaluation of the Casimir energy for such a configuration is the subject of the present study. It is worth stressing that this is only a first step because in the final version ARCHIMEDES experiment will make use of high- $T_{c}$ superconducting oxides with a built-in layered structure, like $\mathrm{YBa}_{2} \mathrm{Cu}_{3} \mathrm{O}_{7-x}$, for which a complete theory is as yet unavailable.

Having this in mind, we start with a thorough analysis of two and three coupled Casimir cavities, made by traditional BCS (low- $T_{c}$ ) superconducting material (niobium), so as to 
deal with relatively manageable and well established formulas. On trying to preserve a macroscopic approach, we limited our study to thicknesses between 10 and $100 \mathrm{~nm}$. In the following, referring to Fig. $1, d_{i}$ is the distance of the $i-t h$ cavity from the $(i-1)-t h$, (thickness of the $i$-th cavity), within the slabs 1,3 and 5 there is vacuum while the zones $0,2,4$ and 6 are made of niobium. The thicknesses of the zones 0 and 6 are assumed to be infinite. Although this choice is dictated by simplicity and by the well-established superconducting properties of $\mathrm{Nb}$, this is a first necessary step to prepare future studies aimed at considering the more elaborate case of high- $T_{c}$ superconductors, as required by the roadmap of the ARCHIMEDES experiment.

Section II studies the Casimir energy of a multilayer cavity, while Sec. III evaluates the Casimir energy in the normal and superconducting phases. Variation of the energy in the transition is obtained in Sec. IV, including a detailed numerical analysis of the Matsubara

zero-mode contribution. Section V extends this scheme to the three-layer configuration, and concluding remarks are made in Sec. VI, while relevant details are given in the Appendices.

\section{THE CASIMIR ENERGY OF A MULTILAYER CAVITY}

As it is customary [7, 8, at finite temperature, the Casimir variation across the transition from a metallic to a superconducting phase is obtained as the difference between the free Casimir energy in the metallic state and the same after the transition to superconducting state takes place: $\delta E(T)=E_{n}-E_{s}$. The energy per unit area of a single cavity, (012) in Fig. 1, can be written, at finite temperature $T$, as the sum of the contributions of the transverse electric $(T E)$ and transverse magnetic $(T M)$ modes (see, for example, [12]) :

$$
E\left[d_{1}, d_{2}\right]=k_{B} T \sum_{l=0}^{\infty} \int \frac{d \mathbf{k}_{\perp}}{(2 \pi)^{2}}\left(\log \Delta^{T E}\left(\xi_{l}\right)+\log \Delta^{T M}\left(\xi_{l}\right)\right)=: \sum_{l=0}^{\infty} E\left[l, d_{1}, d_{2}\right]
$$

where $\xi_{l}=2 \pi l k_{B} T$ are the Matsubara frequencies, $k_{B}$ is the Boltzmann constant, $l=$ $0,1,2, \ldots$, the superscript ' on the sum means that the zero mode must be multiplied by a factor $\frac{1}{2}$,

$$
\Delta_{T M}\left(\xi_{l}\right)=\left(r_{T M}^{0,1}\left(\xi_{l}\right) r_{T M}^{1,2}\left(\xi_{l}\right) e^{-2 d_{1} K_{1}}+1\right), \Delta_{T E}\left(\xi_{l}\right)=\left(r_{T E}^{0,1}\left(\xi_{l}\right) r_{T E}^{1,2}\left(\xi_{l}\right) e^{-2 d_{1} K_{1}}+1\right)
$$

and the reflection coefficients $r_{(T M, T E)}^{i, j}\left(\xi_{l}\right)$ are given by (see [12]):

$$
r_{T M}^{i, j}\left(\xi_{l}\right)=\frac{\epsilon_{i}\left(\xi_{l}\right) K_{j}\left(\xi_{l}\right)-\epsilon_{j}\left(\xi_{l}\right) K_{i}\left(\xi_{l}\right)}{\epsilon_{i}\left(\xi_{l}\right) K_{j}\left(\xi_{l}\right)+\epsilon_{j}\left(\xi_{l}\right) K_{i}\left(\xi_{l}\right)}, r_{T E}^{i, j}\left(\xi_{l}\right)=\frac{K_{j}\left(\xi_{l}\right)-K_{i}\left(\xi_{l}\right)}{K_{j}\left(\xi_{l}\right)+K_{i}\left(\xi_{l}\right)},
$$

with $K_{i}\left(\xi_{l}\right)=\sqrt{k_{\perp}^{2}+\epsilon_{i}\left(\xi_{l}\right) \xi_{l}^{2}}$. We point out that our approach captures the relevant length scale of a superconductor, the London penetration depth $\lambda_{L}$, through the expression of $\epsilon_{i}\left(\xi_{l}\right)$ in terms of the correction to the optical conductivity when entering the superconducting state, $\delta \sigma_{B C S}(i \xi)$, see below and Appendix B. In particular, for $\xi_{l} \rightarrow 0$, we have $\epsilon_{i}\left(\xi_{l}\right) \xi_{l}^{2} \rightarrow \lambda_{L}^{-2}$.

We characterize the properties of the $i-t h$ material trough the dielectric function $\epsilon_{i}\left(\xi_{l}\right)$ and the change in the Casimir energy is given simply by the modification of the $\epsilon\left(\xi_{l}\right)$ due to the transition [7, 8]. As we said, in the following we report calculations for the case in which 


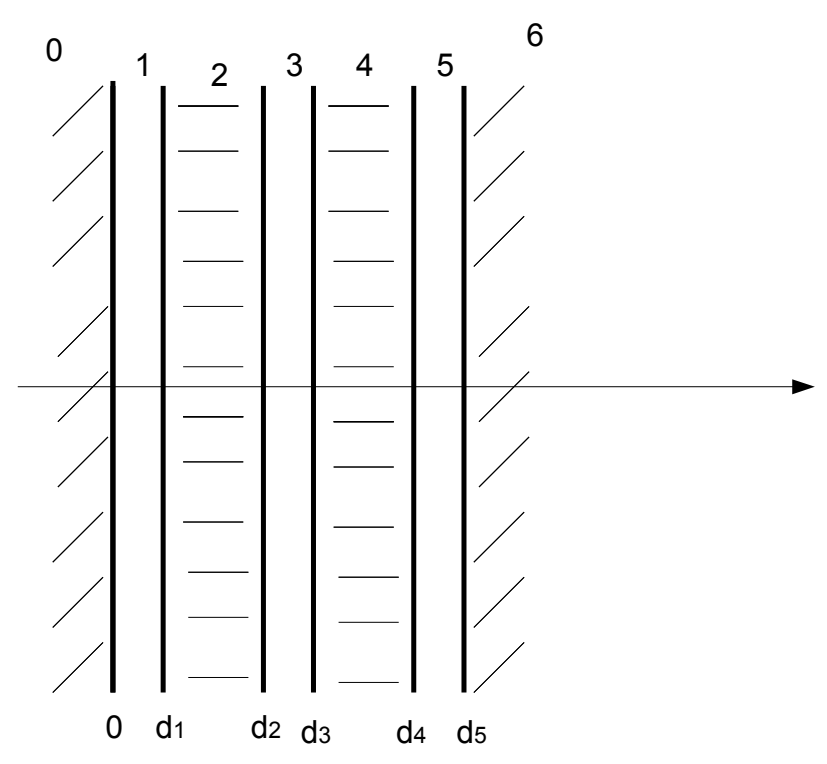

FIG. 1: A three layer cavity. In the $0,2,4$, and 6 zone there is $\mathrm{Nb}$; in the $1,3,5$ vacuum. $d_{i}$ is the thickness of the $i-t h$ slab

the material is $\mathrm{Nb}$ and the spacer is vacuum (the modifications introduced by a dielectric spacer deserve a separate study).

To obtain the formulas for two and three cavities we solve the problem by imposing the continuity of the tangential component of the $\vec{E}$ and $\vec{H}$ fields (non-magnetic media) and the normal component of the $\vec{D}$ and $\vec{B}$ at the interface [13, 14]. Thus, for example, in the case of the three cavities (012-234-456) in Fig. 1 we have that the $\Delta$ functions appearing in (1) are the determinant of the matrix of the coefficients $M_{i j}$ (just to give an idea we report the expression for the $T M$ - modes in appendix A) from which it is possible to extract the case of one, two, and three cavities by taking $(i, j)=1 \ldots 4,(i, j)=1 \ldots 8,(i, j)=1 \ldots 12$, respectively:

$$
\Delta_{(T M, T E)}=\operatorname{det}\left(M_{(T M, T E)}^{i j}\right) .
$$

In the following we will omit the subscript $T M, T E$ if no ambiguity is generated. All the 
formulas for the two cases can be obtained using respectively the $T M$ or $T E$ reflection coefficients. Defining (no summation over repeated indices)

$E^{i j l}=r^{i, j} r^{j, l} e^{-2 d_{j} K_{j}}+1, F^{i j l}=r^{i, j} e^{-2 d_{j} K_{j}}+r^{j, l}, G^{i j l}=r^{i, j}+e^{-2 d_{j} K_{j}} r^{j, l}, H^{i j l}=e^{-2 d_{j} K_{j}}+r^{i, j} r^{j, l}$,

we have for the single cavity (012) in Fig. 1

$$
\Delta^{(1)}=E^{012}
$$

for two cavities $(012-234)$ :

$$
\begin{aligned}
\Delta^{(2)} & =E^{012} E^{234}+e^{-2\left(d_{2} k_{2}\right)} F^{012} G^{234}=: E^{012} E^{234}+I_{012}^{(2)} \quad \text { and } \\
\log \Delta^{(2)} & =\log \left(E^{012} E^{234}\right)+\log \left(1+\frac{I_{012}^{(2)}}{E^{012} E^{234}}\right)
\end{aligned}
$$

and for the three cavities:

$$
\begin{aligned}
\Delta^{(3)}= & E^{012} E^{234} E^{456}+e^{-2\left(d_{2} k_{2}+d_{4} k_{4}\right)} F^{012} H^{234} G^{456} \\
& +e^{-2 d_{2} k_{2}} E^{456} F^{012} G^{234}+e^{-2 d_{4} k_{4}} E^{012} F^{234} G^{456} \\
& =: E^{012} E^{234} E^{456}+I^{(3)}+E^{456} I_{012}^{(2)}+E^{012} I_{234}^{(2)} \\
\log \Delta^{(3)}= & \log \left(E^{012} E^{234} E^{456}\right)+\log \left(1+\frac{I^{(3)}}{E^{012} E^{234} E^{456}}\right) \\
& +\log \left(1+\frac{E^{456} I_{012}^{(2)}+E^{012} I_{234}^{(2)}}{E^{012} E^{234} E^{456}+I^{(3)}}\right)
\end{aligned}
$$

In this way, when $d_{2} \rightarrow \infty$ [see Eq. (3)] $I_{012}^{(2)} \rightarrow 0$ and

$$
\log \Delta^{(2)}=\log E^{012} E^{234}=\log E^{012}+\log E^{234} .
$$

That is to say, when the two cavities are far away their energy is simply the sum of the individual contributions. In this respect the second term on the right of Eq. (3), $I^{(2)}$, can be seen as the energy due to the coupling of the two cavities (012) - (234).

When $d_{1}=d_{3}=d_{5}, d_{2}=d_{4}, \epsilon_{0}=\epsilon_{2}=\epsilon_{4}, \epsilon_{1}=\epsilon_{3}=\epsilon_{5}$ we obtain $E_{T M}^{012}=E^{234}, F^{012}=F^{234}$ so that we can omit the subscripts:

$$
\log \Delta^{(2)}=\log \left(\Delta^{(1)}\right)^{2}+\log \left(1+\frac{I^{(2)}}{\left(\Delta^{(1)}\right)^{2}}\right) .
$$

For the three cavities $(012-234-456)$, formulas are written so as to make evident the contribution to the energy resulting from the sum of the energies of the single cavity, with respect to the one coming from the coupling of the two possible pairs of cavities $(012-234),(234-456)$, and the one coming from the coupling of the three: $I^{(3)}$. Thus, under the previous hypothesis,

$$
\begin{aligned}
\Delta^{(3)} & =\left(\Delta^{(1)}\right)^{3}+2 I^{(2)}+I^{(3)} \text { and we can write } \\
\log \Delta^{(3)} & =\log \left(\Delta^{(1)}\right)^{3}+\log \left(1+2 \frac{I^{(2)}}{\left(\Delta^{(1)}\right)^{3}+I^{(3)}}\right)+\log \left(1+\frac{I^{(3)}}{\left(\Delta^{(1)}\right)^{3}}\right) .
\end{aligned}
$$


In a sense, we are writing the energy as a sum of the energy of the single cavity plus the coupling energy between the nearest neighbor, plus the coupling energy among the second nearest neighbor and so on. In this way we will have a clear indication of the strength of the coupling between the cavities at the various orders. As far as we know this way of displaying the various contribution to the Casimir energy has been obtained for the first time in [15] where the so called TGTG formula ( $T$ being the Lippmann-Schwinger $T$ operator an $G$ the translation matrix), is used, see also [16, 17]. In our case it can be simply recovered by observing that the determinant of a $N \times N$ complex block matrix can be obtained in terms of the determinants of its constituent blocks [18].

The Casimir energy in the superconducting phase is obtained by replacing, in the reflection coefficients, the expression of the dielectric function with the corresponding obtained using the BCS theory [19, 20], see Appendix B. In the following we will characterize the dielectric properties of the material by means of the Drude model (but see conclusions):

$$
\begin{array}{rlrl}
\epsilon(i \xi) & =1+\frac{\sigma(i \xi)}{\xi}, & & \text { with } \\
\sigma_{k}(i \xi) & =\frac{\sigma_{0 k}}{\gamma+\xi}, & & \text { for conducting materials and } \\
\sigma_{k}(i \xi) & =\frac{\sigma_{0 k}}{\gamma+\xi}+\delta \sigma_{B C S}(i \xi), & \text { for superconducting materials }
\end{array}
$$

where the expression of $\delta \sigma_{B C S}(i \xi)$ is given in Appendix B (see [20]).

Thus

$$
\begin{aligned}
\delta E(T) & =E_{n}(T)-E_{s}(T) \\
& =\frac{k T}{2} \sum_{l=0}^{\infty} \int \frac{d \mathbf{k}_{\perp}}{(2 \pi)^{2}}\left(\log \frac{\Delta_{n, T E}^{(k)}}{\Delta_{s, T E}^{(k)}}+\log \frac{\Delta_{n, T M}^{(k)}}{\Delta_{s, T M}^{(k)}}\right)=: \sum_{l=0}^{\infty} \delta E\left[l, d_{1}, d_{2}\right] .
\end{aligned}
$$

where $\Delta_{n, T E, T M}^{(k)}, \Delta_{s, T E, T M}^{(k)}$ are the generating functions (this nomenclature denotes here just the determinant of the matrix whose zeros provide, implicitly, the allowed energies) of the normal and superconducting phases, and, depending on how many cavities we are considering $(1,2$, or 3$)$ we must take $k=1,2$, or 3 respectively.

\section{CASIMIR ENERGY IN THE NORMAL AND SUPERCONDUCTING PHASES}

All results described hereafter are obtained for $\mathrm{Nb}$, and we use the following values for the critical temperature and plasma frequency $T_{c}=9.25 \mathrm{~K}, \hbar \omega_{\text {nio }}=9.268 \mathrm{eV}$ and work at the temperature $T=9.157 \mathrm{~K}$. We start by choosing $d_{1}=300 \mathrm{~nm}$, and $d_{2}=600 \mathrm{~nm}$, so as to have results that can be compared with standard formulas.

We find for the energy in the normal phase $E_{n}$, for fixed $d_{1}, d_{2}$, and different values of number of Matsubara modes $\left(n_{\text {mod }}\right): E_{n}^{n_{\text {mod }}}\left[d_{1}, d_{2}\right]=\sum_{j=0}^{n_{\text {mod }}} E_{n}\left[j, d_{1}, d_{2}\right]$ (N.B. in the following all the quoted numbers that concern energy or difference of energy are in $\left.\frac{\mathrm{J}}{\mathrm{m}^{2}}\right)$ : 


\begin{tabular}{|c|c|}
\hline$n_{\text {mod }}$ & $E_{n}^{n_{\text {mod }}}[300,600] \cdot 10^{8}$ \\
\hline 100 & -1.6577 \\
\hline 200 & -2.2248 \\
\hline 300 & -2.3760 \\
\hline 400 & -2.4122 \\
\hline 500 & -2.4203 \\
\hline 1000 & -2.4226 \\
\hline
\end{tabular}

Thus, at least 500 Matsubara modes are necessary to obtain a result stable at the second decimal digit.

These results can be compared with the approximate result obtained by Bordag et al. [12] $\left(t_{0}=\frac{c \hbar}{2 a k_{B} T}, D_{0}=\frac{c}{a \omega_{p}}\right)$ :

$$
\begin{aligned}
E & =\frac{c \hbar}{8 \pi a^{3}}\left[D_{0}^{2}\left(\frac{3 \zeta(3)}{t_{0}}-\frac{4 \pi^{3}}{25}\right)+D_{0}\left(\frac{2 \pi^{3}}{45}-\frac{\zeta(3)}{t_{0}}\right)+\frac{\zeta(3)}{2 t_{0}}-\frac{\pi^{3}}{90}\right] \\
& =-1.258 \cdot 10^{-8} .
\end{aligned}
$$

As expected, because of the strong suppression of the exponential for large $d_{2}$, the contribution of the coupling term between the two cavities (012)-(123) is about ten orders of magnitude smaller than the energy obtained from each cavity. With

$$
\begin{aligned}
E\left[d_{1}, d_{2}\right] & =\frac{k T}{2} \sum_{l=0}^{\infty} \int \frac{d \mathbf{k}_{\perp}}{(2 \pi)^{2}}\left[\log \left(E^{012} E^{234}\right)+\log \left(1+\frac{I_{012}^{(2)}}{E^{012} E^{234}}\right)\right]_{T M}+[T E] \\
& =: E_{T M}^{(2)}\left[d_{1}, d_{2}\right]+C_{T M}^{(2)}\left[d_{1}, d_{2}\right]+E_{T E}^{(2)}\left[d_{1}, d_{2}\right]+C_{T E}^{(2)}\left[d_{1}, d_{2}\right] \\
& =: E^{(2)}\left[d_{1}, d_{2}\right]+C^{(2)}\left[d_{1}, d_{2}\right]
\end{aligned}
$$

we obtain $\left(n_{\text {mod }}=500\right)$ :

$$
E_{n}^{(2)}[300,600]=-2.42035 \cdot 10^{-8} ; \quad C_{n}^{(2)}[300,600]=-2.30743 \cdot 10^{-17} .
$$

Indeed, having $d_{1} \ll d_{2}$, the total energy is simply the sum of the energies of the two cavities:

$$
\frac{E_{n}{ }^{(2)}[300,600]}{2}=\frac{-2.42035}{2} \cdot 10^{-8}=-1.2102 \cdot 10^{-8} \approx-1.258 \cdot 10^{-8}
$$

In the superconducting phase we have, more or less, the same behavior:

\begin{tabular}{|c|c|}
\hline$n_{\text {mod }}$ & $E_{s}^{n_{\text {mod }}}[300,600] \cdot 10^{8}$ \\
\hline 100 & -1.6584 \\
\hline 200 & -2.2255 \\
\hline 300 & -2.3767 \\
\hline 400 & -2.4129 \\
\hline 500 & -2.4210 \\
\hline 1000 & -2.4232 \\
\hline
\end{tabular}

The Casimir energy is always greater than the corresponding energy in the normal phase so that, as expected, the difference is always positive. Once more, the contribution from 
the energy of the two cavities is much larger than the contribution from the coupling, but "only" by about four orders of magnitude:

$E_{s}^{500}[300,600]=-2.4210 \cdot 10^{-8}, E_{s}^{(2)}[300,600]=-2.4208 \cdot 10^{-8}, C_{s}^{(2)}[300,600]=-2.0979 \cdot 10^{-12}$.

\section{VARIATION OF THE ENERGY ACROSS THE TRANSITION}

In computing the difference in energy between the two phases, we find that a few tens (50) of modes are sufficient to obtain good values. This is a consequence of the fact that the high-energy part of the spectrum is essentially the same in the metal and in the superconductor, making the energy difference a quantity that converges much more rapidly than the individual terms, as a function of the upper cutoff in the Matsubara frequency, $n_{\text {mod }}$.

On defining $\delta E^{(2)}+\delta C^{(2)}$, i.e.

$$
\delta E^{n_{m o d}}\left[d_{1}, d_{2}\right]=E_{n}{ }^{(2)}\left[d_{1}, d_{2}\right]-E_{s}^{(2)}\left[d_{1}, d_{2}\right]+C_{n}{ }^{(2)}-C_{s}^{(2)}=: \delta E^{(2)}+\delta C^{(2)}
$$

as the difference between the terms coming from the energy of the two cavities in the normal and superconducting phase, plus the difference between the values of the coupling in the two phases respectively, we have:

\begin{tabular}{|c|c|c|c|}
\hline$n_{\text {mod }}$ & $\delta E^{n_{\text {mod }}}[300,600] \cdot 10^{12}$ & $\delta E^{(2)} \cdot 10^{12}$ & $\delta C^{(2)} \cdot 10^{12}$ \\
\hline 10 & 6.54447 & 4.44655 & 2.09792 \\
\hline 30 & 6.55295 & 4.45504 & 2.09792 \\
\hline 50 & 6.55406 & 4.45614 & 2.09792 \\
\hline 100 & 6.55461 & 4.45669 & 2.09792 \\
\hline
\end{tabular}

We note that $\delta E^{(2)}$ is of the same order of magnitude of $\delta C^{(2)}$, and when $d_{1}=d_{2} \quad \delta C^{(2)}$ is about two orders of magnitude larger:

$$
\begin{aligned}
& \delta E^{50}[50,50]=1.11259 \cdot 10^{-9}=1.24592 \cdot 10^{-11}+1.10013 \cdot 10^{-9} \\
& \delta E^{50}[10,10]=9.3812 \cdot 10^{-9}=2.34485 \cdot 10^{-11}+9.35775 \cdot 10^{-9} .
\end{aligned}
$$

\section{IV.1. The Matsubara zero-mode contribution}

It turns out that this unexpected behavior is due to the contribution from the Matsubara zero mode. This is evident in the following table where we report, for the $n-t h$ Matsubaramode, the values of the Casimir energy in the normal and superconducting phase, and their difference $\left(d_{1}=d_{2}=100 \mathrm{~nm}\right)$ : 


\begin{tabular}{|c|c|c|c|c|}
\hline$n$ & $E[n, 100,100]=$ & $\left\{E^{(2)}+C^{(2)}\right\}_{T E}$ & $\left\{E^{(2)}+C^{(2)}\right\}_{T M}$ & $\left\{E^{(2)}+C^{(2)}\right\}_{T E+T M}$ \\
\hline \multirow{4}{*}{0} & $E_{n}$ & $0+0$ & $-6.039 \cdot 10^{-10}-2.175 \cdot 10^{-13}$ & $-6.041 \cdot 10^{-10}$ \\
\cline { 2 - 5 } & $E_{s}$ & $-6.284 \cdot 10^{-12}-1.647 \cdot 10^{-13}$ & $-6.039 \cdot 10^{-10}-2.692 \cdot 10^{-10}$ & $-8.795 \cdot 10^{-10}$ \\
\cline { 2 - 5 } & $\delta E$ & $6.284 \cdot 10^{-12}+1.647 \cdot 10^{-13}$ & $0.0+2.690 \cdot 10^{-10}$ & $2.754 \cdot 10^{-10}$ \\
\hline \multirow{4}{*}{1} & $E_{n}$ & $-2.076 \cdot 10^{-10}-6.060 \cdot 10^{-12}$ & $-1.207 \cdot 10^{-9}-1.235 \cdot 10^{-10}$ & $-1.545 \cdot 10^{-9}$ \\
\cline { 2 - 5 } & $E_{s}$ & $-2.087 \cdot 10^{-10}-6.048 \cdot 10^{-12}$ & $-1.207 \cdot 10^{-9}-1.223 \cdot 10^{-10}$ & $-1.545 \cdot 10^{-9}$ \\
\hline \multirow{4}{*}{10} & $\delta E$ & $1.161 \cdot 10^{-12}-1.228 \cdot 10^{-14}$ & $9.480 \cdot 10^{-16}-1.235 \cdot 10^{-12}$ & $-8.536 \cdot 10^{-14}$ \\
\cline { 2 - 5 } & $E_{n}$ & $-4.64934 \cdot 10^{-10}-8.59787 \cdot 10^{-13}$ & $-1.19878 \cdot 10^{-9},-5.58978 \cdot 10^{-12}$ & $-1.67017 \cdot 10^{-9}$ \\
\hline \multirow{4}{*}{50} & $E_{s}$ & $-4.64952 \cdot 10^{-10}-8.5958 \cdot 10^{-13}$ & $-1.19878 \cdot 10^{-9}-5.58814 \cdot 10^{-12}$ & $-1.67018 \cdot 10^{-9}$ \\
\cline { 2 - 5 } & $\delta E$ & $1.80615 \cdot 10^{-14}-2.07135 \cdot 10^{-16}$ & $2.31737 \cdot 10^{-16}-1.64377 \cdot 10^{-15}$ & $1.64423 \cdot 10^{-14}$ \\
\cline { 2 - 5 } & $E_{n}$ & $-5.11734 \cdot 10^{-10}-1.87681 \cdot 10^{-13}$ & $-1.1032 \cdot 10^{-9}-9.34075 \cdot 10^{-13}$ & $-1.61606 \cdot 10^{-9}$ \\
\hline & $E_{s}$ & $-5.11735 \cdot 10^{-10}-1.8768 \cdot 10^{-13}$ & $-1.1032 \cdot 10^{-9}-9.34068 \cdot 10^{-13}$ & $-1.61606 \cdot 10^{-9}$ \\
\hline
\end{tabular}

TABLE I: Contributions of the TE and TM modes for different values of $n$

and summing the first 50 modes:

$$
\delta E^{50}[100,100]=2.76004 \cdot 10^{-10}=8.54145 \cdot 10^{-12}+2.67463 \cdot 10^{-10} .
$$

A close look at the table makes it evident that the result is almost completely due to the contribution of the coupling term of the zero mode. Indeed, $C_{s, T M}^{(2)}$ is about 3 orders of magnitude larger than the corresponding in the normal case $C_{n, T M}^{(2)}$ while all the other terms are of the same order of magnitude (in some case egual) so that in the difference they cancel each other.

\section{ENERGY OF THE THREE-LAYER CONFIGURATION}

The behavior discussed in the previous section is confirmed for the three-layer configuration:

$$
\begin{aligned}
E\left[d_{1}, d_{2}, d_{4}\right]= & \frac{k_{B} T}{2} \sum_{l=0}^{\infty} \int \frac{d \mathbf{k}_{\perp}}{(2 \pi)^{2}}\left[\log \left(E^{012} E^{234} E^{456}\right)+\log \left(1+\frac{I^{(3)}}{E^{012} E^{234} E^{456}}\right)\right. \\
& \left.+\log \left(1+\frac{E^{456} I_{012}^{(2)}+E^{012} I_{234}^{(2)}}{E^{012} E^{234} E^{456}+I^{(3)}}\right)\right]_{T M}+[T E], \\
= & : E^{(3)}\left[d_{1}, d_{2}, d_{4}\right]+C^{(3)}\left[d_{1}, d_{2}, d_{4}\right]+C^{(2)}\left[d_{1}, d_{2}, d_{4}\right] \\
E^{n_{\text {mod }}}\left[d_{1}, d_{2}, d_{4}\right]:= & \sum_{l=0}^{n_{\text {mod }}} E\left[l, d_{1}, d_{2}, d_{4}\right],
\end{aligned}
$$

To have a comparison between the formulae for two and three cavities let us compute the Casimir energy for the three layer when $d_{4}$ is very large. In this case, since the third cavity is distant from the other two, it decouples and the result would be the sum of the energy of 
a double cavity plus the energy of a third one. Indeed we find:

\begin{tabular}{|c|c|c|c|}
\hline$E_{n}^{500}(100,100,300)$ & $E_{n}^{(3)}$ & $C_{n}^{(2)}$ & $C_{n}^{(3)}$ \\
\hline$-6.2636 \cdot 10^{-7}$ & $-6.2574 \cdot 10^{-7}$ & $-6.2390 \cdot 10^{-10}$ & $6.73512 \cdot 10^{-15}$ \\
\hline
\end{tabular}

this is exactly three halves the energy of a double cavity:

$$
E_{n}^{500}(100,100)=-4.17785 \cdot 10^{-7} \approx-\frac{2}{3} 6.26365 \cdot 10^{-7}=4.17577 \cdot 10^{-7},
$$

as expected. Of course, this is a consequence of the strong exponential suppression present in this term, see the expression of $I^{(3)}$ in Eq.(5). Taking $d_{4}=d_{1}=d_{2}=100 \mathrm{~nm}$ we find

\begin{tabular}{|c|c|c|c|}
\hline$E_{n}^{500}(100,100,100)$ & $E_{n}^{(3)}$ & $C_{n}^{(2)}$ & $C_{n}^{(3)}$ \\
\hline$-6.26989 \cdot 10^{-7}$ & $-6.25742 \cdot 10^{-7}$ & $-1.25193 \cdot 10^{-9}$ & $4.39881 \cdot 10^{-12}$ \\
\hline
\end{tabular}

thus the contribution due to the coupling of the three cavities is three orders of magnitude larger than in the previous case but still, for the normal Casimir energy, very much smaller than the sum of the energies of the three individual cavities.

Once again, things are different when computing the difference between the energy in the normal and superconducting phase. Indeed, in this case the contribution from the $n-t h$ mode is, with obvious significance for the indicated symbols:

\begin{tabular}{|c|c|c|c|c|}
\hline$n$ & $\delta E(n, 100,100,100)$ & $\delta E^{(3)}$ & $\delta C^{(2)}$ & $\delta C^{(3)}$ \\
\hline 0 & $5.47751 \cdot 10^{-10}$ & $9.42558 \cdot 10^{-12}$ & $5.84966 \cdot 10^{-10}$ & $-4.66401 \cdot 10^{-11}$ \\
\hline 1 & $-7.53124 \cdot 10^{-13}$ & $1.74333 \cdot 10^{-12}$ & $-2.57231 \cdot 10^{-12}$ & $7.58578 \cdot 10^{-14}$ \\
\hline 10 & $2.3738 \cdot 10^{-14}$ & $2.7440 \cdot 10^{-14}$ & $-3.7044 \cdot 10^{-15}$ & $2.49605 \cdot 10^{-18}$ \\
\hline 100 & $8.97745 \cdot 10^{-17}$ & $9.15143 \cdot 10^{-17}$ & $-1.73989 \cdot 10^{-18}$ & $9.25498 \cdot 10^{-23}$ \\
\hline
\end{tabular}

We immediately realize that even in this case the energy is due almost completely to the coupling of nearest cavities $\delta C^{(2)}$. Note that the $\delta C^{(3)}$ term is about one order of magnitude smaller than the corresponding $\delta C^{(2)}$. Summing on the first $n$ modes we find

\begin{tabular}{|c|c|c|c|c|}
\hline$n_{\text {mod }}$ & $\delta E^{n_{\text {mod }}}(100,100,100)$ & $\delta E^{(3)}$ & $\delta C^{(2)}$ & $\delta C^{(3)}$ \\
\hline 10 & $5.47634 \cdot 10^{-10}$ & $1.2644 \cdot 10^{-11}$ & $5.81547 \cdot 10^{-10}$ & $-4.6557 \cdot 10^{-11}$ \\
\hline 50 & $5.47789 \cdot 10^{-10}$ & $1.28122 \cdot 10^{-11}$ & $5.81533 \cdot 10^{-10}$ & $-4.6557 \cdot 10^{-11}$ \\
\hline 100 & $5.47800 \cdot 10^{-10}$ & $1.28236 \cdot 10^{-11}$ & $5.81533 \cdot 10^{-10}$ & $-4.6557 \cdot 10^{-11}$ \\
\hline
\end{tabular}

For layers $10 \mathrm{~nm}$ thick we find:

\begin{tabular}{|c|c|c|c|}
\hline$E_{n}^{500}(10,10,10)$ & $E_{n}^{(3)}$ & $C_{n}^{(2)}$ & $C_{n}^{(3)}$ \\
\hline$-1.134 \cdot 10^{-4}$ & $-7.880 \cdot 10^{-5}$ & $-3.641 \cdot 10^{-5}$ & $1.761 \cdot 10^{-6}$ \\
\hline
\end{tabular}

and

\begin{tabular}{|c|c|c|c|}
\hline$\delta E^{100}(10,10,10)$ & $\delta E^{(3)}$ & $\delta C^{(2)}$ & $\delta C^{(3)}$ \\
\hline $1.875 \cdot 10^{-8}$ & $3.517 \cdot 10^{-11}$ & $2.166 \cdot 10^{-8}$ & $-2.946 \cdot 10^{-9}$ \\
\hline
\end{tabular}



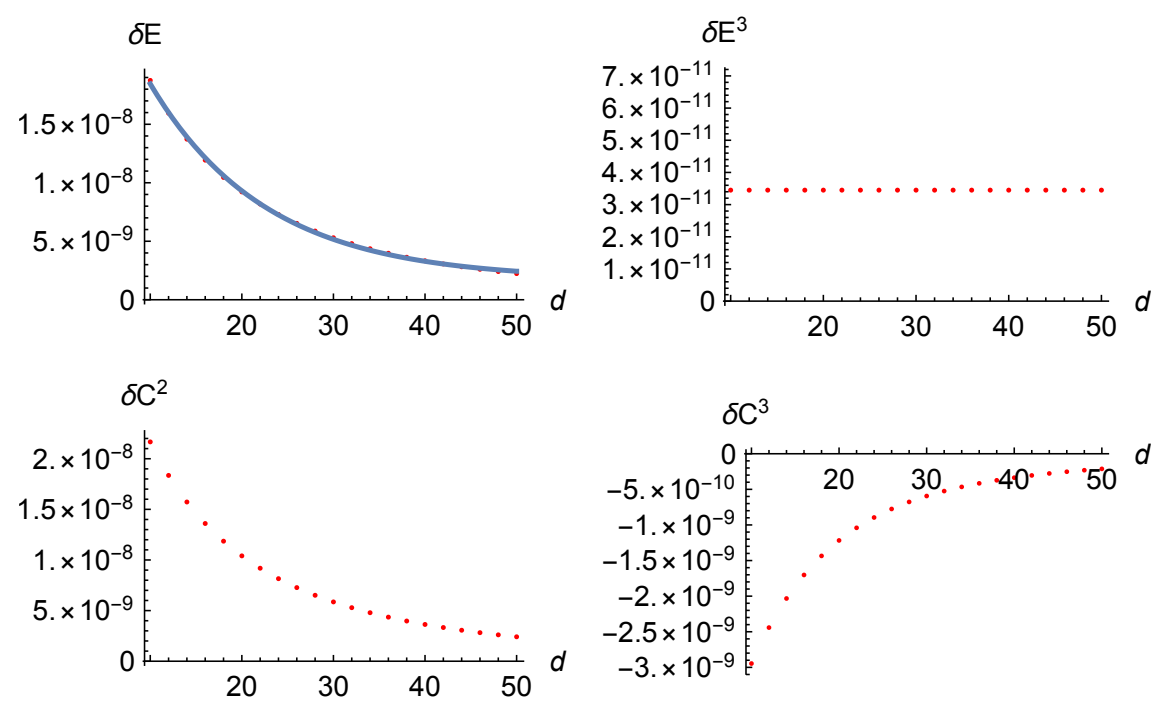

FIG. 2: The behavior with respect to $d_{2}=d_{4}=d \in[10,50] n m$ of the Casimir energy $\delta E$ and of the various components $\delta E^{3}, \delta C^{2}$, and $\delta C^{3}$ for the three-layer configuration with $d_{1}=10 \mathrm{~nm}$. In the plot of $\delta E$ it is shown, also, the fitting curve.

To give an idea of the dependence of the Casimir energy on the parameters $d_{1}, d_{2}, d_{4}$, we show in the figure 2 the contribution of the three terms $\delta E^{3}, \delta C^{2}$, and $\delta C^{3}$ to the energy difference between the normal and the superconducting phase, $\delta E$, with respect to $d_{2}=d_{4}=$ $d \in[10,50] \mathrm{nm}$ with $d_{1}=10 \mathrm{~nm}$. In blue it is shown a fit of $\delta E$ obtained by means of the function $\delta E=a+b e^{-\left(\frac{x}{x_{0}}\right)}$ with $a=1.73 \cdot 10^{-9} \mathrm{~J} / \mathrm{m}^{2}, b=3.69 \cdot 10^{-8} \mathrm{~J} / \mathrm{m}^{2}, x_{0}=12.63 \mathrm{~nm}$. Note that the red dots are completely covered by the fitting curve. The only term that substantially depends on $d_{1}$ is $\delta E^{3}$, i.e. the sum of the energies of the single cavity whose thickness is $d_{1}$. On the contrary the other terms almost depend on $d_{2}, d_{4}$ exclusively. Being $\delta E^{3}$ very much smaller than $\delta C^{2}$ and $\delta C^{3}$, this fit is very stable with respect the variation of $d_{1}$ see FIG. 3 where the same fitting curve is overimposed on the data relative to $d_{1}=500 \mathrm{~nm}$

We conclude that, the contribution from the coupling of the three cavities being so large: $\delta C^{3}$ can turn out to be only one order of magnitude smaller than $\delta C^{2}$, it will be therefore necessary to analyze the situation of four coupled cavities.

Some comments about the contribution of the $T M$ zero mode are in order at this point (in the following we will analyze the configuration of two coupled cavities but the generalization to three is straightforward). In the $\xi \mapsto 0$ limit, assuming vacuum between the two superconducting layers (see appendix B), one obtains $r_{T M}^{i, j}=1$ for both the normal and superconducting case, so that:

$$
\begin{aligned}
E_{T M, n}^{(2)} & =E_{T M, s}^{(2)}=\frac{k_{B} T}{4} \int \frac{d \mathbf{k}_{\perp}}{(2 \pi)^{2}} \log \left(1-e^{-2 d_{1} K_{1}}\right) \\
C_{T M, n}^{(2)} & =\frac{k_{B} T}{4} \int \frac{d \mathbf{k}_{\perp}}{(2 \pi)^{2}} \log \left(1-e^{-2 d_{2} K_{0, n}}\right) \\
C_{T M, s}^{(2)} & =\frac{k_{B} T}{4} \int \frac{d \mathbf{k}_{\perp}}{(2 \pi)^{2}} \log \left(1-e^{-2 d_{2} K_{0, s}}\right)
\end{aligned}
$$




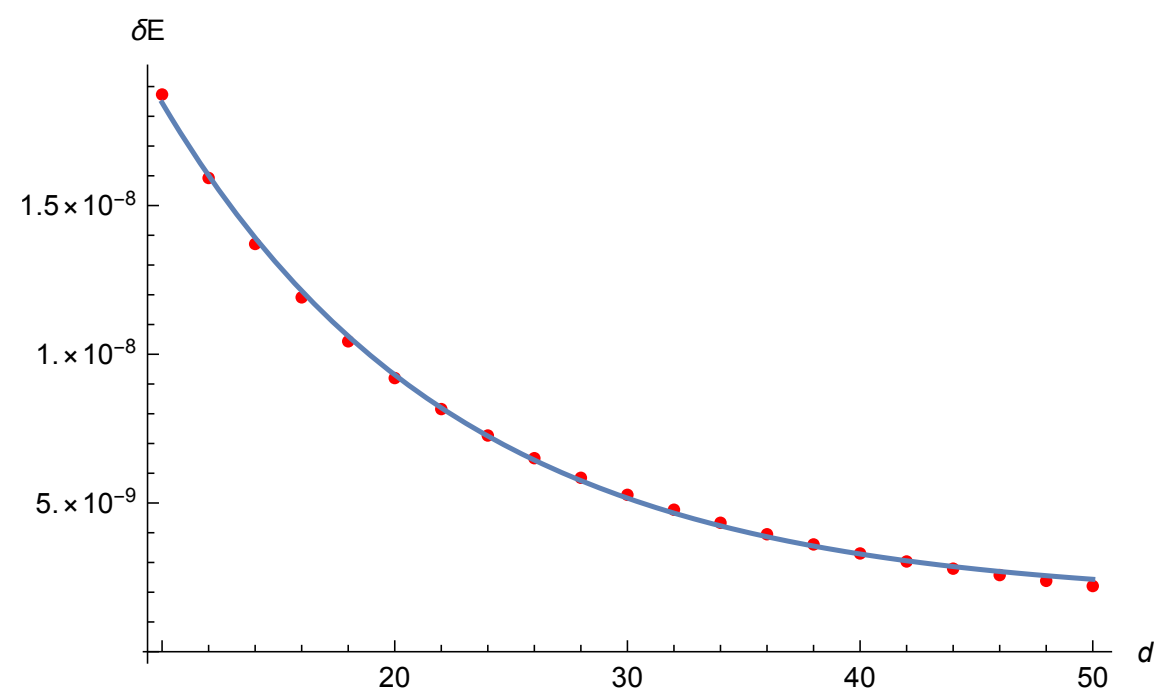

FIG. 3: The behavior with respect to $d_{2}=d_{4}=d \in[10,50] \mathrm{nm}$ of the Casimir energy $\delta E$ for the three-layer configuration with $d_{1}=500 \mathrm{~nm}$ and the fitting curve with the parameters obtained for the case $d_{1}=10 \mathrm{~nm}$.

where $d_{i}$ are measured in $\mathrm{nm}, K_{i}$ in $\mathrm{nm}^{-1}$, and $K_{i, n / s}=\sqrt{k_{\perp}^{2}+\alpha_{i, n / s}^{2}}$. We immediately realize that the contribution of the energies of the two cavities $E_{T M}^{(2)}$ is exactly the same in the normal and in the superconducting phase so that they cancel in the difference. On the contrary the contribution of the interaction terms $C_{T M}^{(2)}$ in the two phases are different thanks to the presence of $K_{0, n}$ and $K_{0, s}$ respectively. (Note that the dependence of these two terms on $d_{1}$ cancels). Naturally, the strong suppression due to the exponential ensures that the main contribution to the integral comes from small wave numbers $k_{\perp}$ (hereafter measured in $\left.\mathrm{nm}^{-1}\right)$, taking $d_{1}=d_{2}=100 \mathrm{~nm}$ and $\alpha_{1}^{2}:=\sqrt{k_{\perp}^{2}+\left(\frac{\omega_{\text {nio }}}{c}\right)^{2}}=\sqrt{k_{\perp}^{2}+0.00221} \mathrm{~nm}^{-1}$, $\alpha_{0, n}^{2}:=k_{\perp}^{2}$, and $\alpha_{0, s}^{2}:=\sqrt{k_{\perp}^{2}+\left(\frac{\omega_{s}}{c}\right)^{2}}=\sqrt{k_{\perp}^{2}+4.306 \cdot 10^{-6}} \mathrm{~nm}^{-1}$, we recover the numbers in Table I. Of course the huge difference reduce drastically for smaller values of $d_{2}$ because smaller values of $d_{2}$ allow for the contribution to the integral from larger values of $k_{\perp}$ so that the dependence on $\alpha_{i, n / s}^{2}$ is less evident. For example with $d_{2}=10 \mathrm{~nm}$ we get $C_{T M, n}^{(2)}=1.78 \cdot 10^{-8} \mathrm{~J}$ and $C_{T M, s}^{(2)}=-3.011 \cdot 10^{-8} \mathrm{~J}$. However in this case, even though the two terms are very much closer, their absolute value is larger so that they still give a strong contribution to the energy, thus the values of $\delta E$ is large, see Eqs. (12) and (13). We expect that this behavior could change when a dielectric is inserted between the two layers.

\section{CONCLUDING REMARKS}

In this paper we performed a series of numerical calculations aimed at the computation of the Casimir energy in the normal and superconducting phase for a multilayered cavity. This is of particular interest for the ARCHIMEDES experiment aimed at weighing the vacuum energy of a multi-cavity by modulating the reflectivity of the constituting plates from the metallic to the superconducting phase. As pointed out in [1] with a single cavity and with a standard BCS superconductor a ratio $\eta=\frac{\Delta E_{c a s}}{E_{c a s}} \sim 10^{-8}$ is expected. For this value there would 
be no possibility for the experiment to detect the signal. However, and quite surprisingly, our results are orders of magnitude larger: We obtained a very large contribution from a term resulting from the coupling of nearest neighbor cavities in the superconducting phase. This strong enhancement of $\eta$ results from the use of a superconducting multi-layer (at least two) structure and it can be attributed to the strong contribution of the $T M$ Matsubara zero mode. From the point of view of the experiment these results are quite promising.

The important role played by the static TM physically arises because, while a static electric field in a superconductor (and in a metal as well) is rapidly screened on short length-scales, the magnetic field parallel to the vacuum-Nb interface can penetrate over a substantial distance, set by the London penetration depth. This length is shortest in clean $\mathrm{Nb}$, but is still of the order of tens of $\mathrm{nm}$, and increases in the presence of impurities. It is not surprising therefore, that the zero-frequency TM mode links the various adjacent cavities, providing a substantial inter-cavity contribution to the Casimir energy. Therefore, in computing the Casimir energy of a large number of overlapping cavities, it is necessary to take into account the contribution from the coupling of pairs of cavities that can lead to a strong enhancement of the effect. This behavior is confirmed in the case of a three-layer configuration where, in addition, the contribution of the coupling of the three cavities turns out to be about one order of magnitude smaller. At this stage we plan to obtain in a future work an estimate of the contribution of (at least) four coupled cavities. Because of the strong contribution of the zero mode we expect to be able to discriminate between the Drude or plasma model in computing the zero-mode contribution for the Casimir energy. We wish to point out that, even though these results are encouraging, the shift in energy is still not as large as needed. Indeed (see [1], and Refs. therein), to extract the signal we need an energy shift of the order of few joules. With this kind of configuration, even using a very thin layer, of the order of few nanometers, the energy shift is relatively small: $\delta E^{100}(1,1,1)=6.371 \cdot 10^{-8}$ This is a consequence of the smooth dependence of $\delta E$ on $d_{2}$. Indeed, for $d_{2} \leq 10 \mathrm{~nm}$, it can be fitted as (see [8]):

$$
\delta E^{100}\left(10, d_{2}, d_{2}\right)=\frac{\delta E_{0}}{1+\left(\frac{d_{2}}{D}\right)^{s}}
$$

with $\delta E_{0}=9.29 \cdot 10^{-8}, s=0.92, D=2.30 \mathrm{~nm}$. Thus, in this way we can gain at most one order of magnitude. This result strongly support our idea of obtaining such an improvement by using high-temperature superconducting oxides, like $\mathrm{YBa}_{2} \mathrm{Cu}_{3} \mathrm{O}_{7-x}$. In this case, in fact, larger areas can be used (two orders of magnitude), a larger number of layer, $\sim 10^{6}$, can be assembled together, relying on the fine built-in layered structure of cuprates, with thickness of the order of $1 \mathrm{~nm}$. It is possible to work at high temperature, $\sim 100 \mathrm{~K}$ (gaining here a factor ten), and, possibly, other two order of magnitude can be gained from $\Delta T$. Of course, this prevision can prove to be too optimistic and for this reason the extension of the present analysis to such a situation is underway.

\section{ACKNOWLEDGMENTS}

G.E. and C.S. are grateful to the Department of Physics "Ettore Pancini" of Federico II University, Naples, for hospitality and support. 


\section{Appendix A:}

For the case of the $T M$-modes the matching conditions give the following $12 \times 12$ matrix of coefficients:

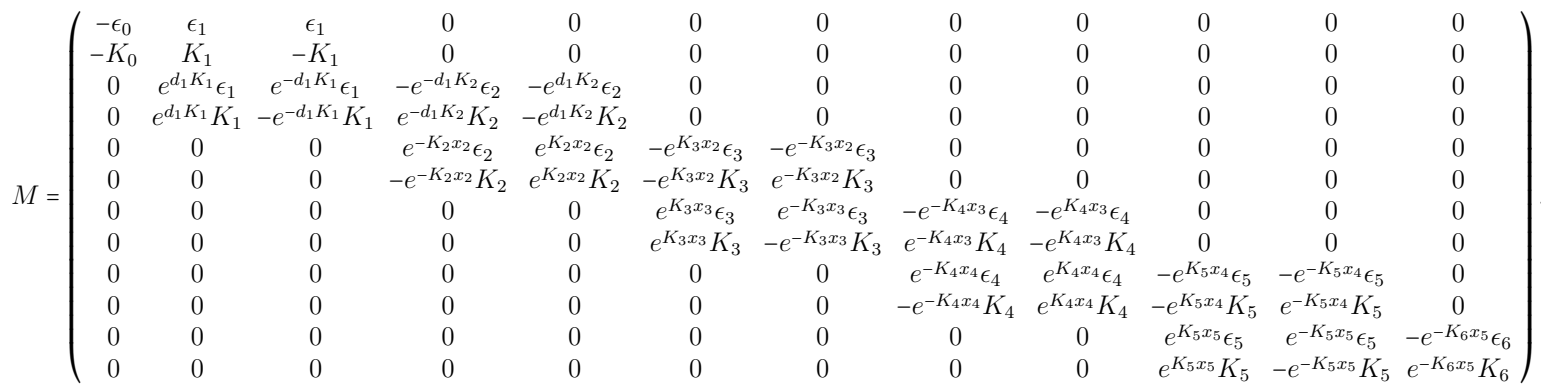

Computing the determinant of the minors of dimensions 4, 8, and 12 respectively we obtain Eqs. (23) 5 ).

\section{Appendix B:}

On writing

$$
\epsilon(i \xi)=1+\frac{\sigma(i \xi)}{\xi}
$$

where $\sigma(i \xi)$ is the conductivity along the imaginary frequencies, we will obtain the dielectric function in the Drude model for the normal case simply by taking

$$
\sigma(i \xi)=\frac{\omega_{p}^{2} / 4 \pi}{\gamma+\xi}
$$

with $\omega_{p}^{2}=4 \pi n e^{2} / m$ the plasma frequency and $\gamma$ the relaxation parameter. While in the superconducting phase the conductivity can be written as [20]

$$
\sigma(i \xi)=\frac{\omega_{p}}{\gamma+\xi}+\delta \sigma_{B C S}(i \xi)
$$

where the correction within the $B C S$ model is given by (in the following $\hbar=1$ )

$$
\begin{aligned}
\delta \sigma_{B C S}(i \xi) & =\frac{\sigma_{0} \gamma}{\xi} \int_{-\infty}^{+\infty} \tanh \left(\frac{E}{2 T}\right) \operatorname{Re}\left[G_{+}(i \xi, \eta)\right] \frac{d \eta}{E}, \\
G_{+}(z, \eta) & =\frac{\eta^{2} Q_{+}(z, E)+A_{+}(z, E)\left(Q_{+}(z, E)+i \gamma\right)}{Q_{+}(z, E)\left[\eta^{2}-\left(Q_{+}(z, E)+i \gamma\right)^{2}\right.}, \\
A_{+}(z, E) & =E(E+z)+\Delta^{2}, \\
Q_{+}^{2}(z, E) & =(E+z)^{2}-\Delta^{2} \\
E & =\sqrt{\eta^{2}+\Delta^{2}} .
\end{aligned}
$$


To obtain the reflection coefficients for the zero mode we have to compute the limit $\xi \rightarrow 0$. In this way, considering that in the 1,3 , and 5 regions there is vacuum, we find

$$
\lim _{\xi \rightarrow 0} r_{T M}^{i, j}(i \xi)=1, \quad \lim _{\xi \rightarrow 0} r_{T E}^{i, j}(i \xi)=0
$$

in the conducting phase. Noting that, when $\xi \rightarrow 0, \delta \sigma_{B C S}(z)$ can be approximated by [20]

$$
\delta \sigma_{B C S}(i \xi) \approx \omega_{s}^{2} / \xi
$$

with

$$
\omega_{s}^{2}=\frac{\omega_{p}^{2}}{\gamma}\left(\pi \Delta \tanh \frac{\Delta}{2 k_{b} T}-4 \gamma \Delta^{2} \int_{0}^{\infty} \frac{\tanh \frac{\sqrt{\Delta^{2}+x^{2}}}{2 k_{b} T}}{\sqrt{\Delta^{2}+x^{2}}\left(\gamma^{2}+x^{2}\right)} d x\right),
$$

we obtain for $r_{T M, T E}^{i, j}$ in the superconducting phase:

$$
\lim _{\xi \rightarrow 0} r_{T M}^{i, j}(i \xi)=1, \quad \lim _{\xi \rightarrow 0} r_{T E}^{i, j}(i \xi)=\frac{k_{\perp}-\sqrt{k_{\perp}^{2}+\omega_{s i}^{2}}}{k_{\perp}+\sqrt{k_{\perp}^{2}+\omega_{s i}^{2}}} .
$$

[1] E. Calloni, M. De Laurentis, R. De Rosa, F. Garufi, L. Rosa, L. Di Fiore, G. Esposito, C. Rovelli, P. Ruggi, and F. Tafuri, Towards weighing the condensation energy to ascertain the Archimedes force of vacuum, Phys. Rev. D 90 (2014) no.2, 022002

[2] G. Bimonte, E. Calloni, G. Esposito and L. Rosa, Energy-momentum tensor for a Casimir apparatus in a weak gravitational field, Phys. Rev. D 74 (2006) 085011 Erratum: Phys. Rev. D 75 (2007) 049904 Erratum: Phys. Rev. D 75 (2007) 089901 Erratum: Phys. Rev. D 77 (2008) 109903

[3] S. A. Fulling, K. A. Milton, P. Parashar, A. Romeo, K. V. Shajesh, and J. Wagner, How does Casimir energy fall?, Phys. Rev. D 76, 025004 (2007).

[4] G. Bimonte, G. Esposito and L. Rosa, From Rindler space to the electromagnetic energymomentum tensor of a Casimir apparatus in a weak gravitational field, Phys. Rev. D 78 (2008) 024010

[5] G. Esposito, G. M. Napolitano and L. Rosa, Energy-momentum tensor of a Casimir apparatus in a weak gravitational field: Scalar case, Phys. Rev. D 77 (2008) 105011

[6] H. B. G. Casimir, Introductory remarks on quantum electrodynamics, Physica 19, 846 (1953).

[7] G. Bimonte, E. Calloni, G. Esposito, L. Milano and L. Rosa, Towards measuring variations of Casimir energy by a superconducting cavity, Phys. Rev. Lett. 94 (2005) 180402

[8] G. Bimonte, E. Calloni, G. Esposito and L. Rosa, Variations of Casimir energy from a superconducting transition, Nucl. Phys. B 726 (2005) 441

[9] G. Bimonte, D. Born, E. Calloni, G. Esposito, U. Huebner, E. Il'ichev, L. Rosa, F. Tafuri, and R. Vaglio: "Low noise cryogenic system for the measurement of the Casimir energy in rigid cavities" J. Phys. A 41, 164023 (2008).

[10] A. Allocca, G. Bimonte, D. Born, E. Calloni, G. Esposito, U. Huebner, E. Il'ichev, L. Rosa, 
and F. Tafuri: "Results of measuring the influence of Casimir energy on superconducting phase transitions" Jour. Super. and Novel Mag. 25, 2557 (2012).

[11] A. Kempf, J. Phys. A 41, 164038 (2008).

[12] M. Bordag, G.L. Klimtchisktaya, U. Mohideen, and V.M. Mostepanenko, Advances in the Casimir Effect (Oxford University Press, Oxford, 2009).

[13] M. Bordag, U. Mohideen and V.M. Mostepanenko, New developments in the Casimir effect, Phys. Rep. 353, 1 (2001).

[14] J. D. Jackson, Classical Electrodynamics (John Wiley \& Sons, New-York, 1998).

[15] O. Kenneth, and I. Klich, Phys. Rev. Lett. 97, 160401 (2006).

[16] K.V. Shajesh, and M. Schaden, Phys. Rev. D 83, 125032 (2011).

[17] L.P. Teo, Int. J. Mod. Phys. A 27, 1230021 (2012).

[18] J.R. Silvester, Math. Gaz., 84, 460 (2000); P. D. Powell, arXiv:1112.4379 [math.RA] (2011)

[19] W. Zimmermann, E.H. Brandt, M. Bauer, E. Seider, and L. Genzel, Optical conductivity of BCS superconductors with arbitrary purity, Physica C 183, 99 (1991).

[20] G. Bimonte, H. Haakh, C. Henkel, and F. Intravaia, Optical BCS conductivity at imaginary frequencies and dispersion energies of superconductors, J. Phys. A 43, 145304 (2010).

[21] G. Barton, J. Phys. A 38, 2997 (2005). 